\title{
Médiévales
}

Langues, Textes, Histoire

\section{Abolala SOUDAVAR, The Aura of Kings, Legitimacy and Divine Sanction in Iranian Kingship, Mazda Publisher, Costa Mesa, 2003, 178 p.}

\section{Anna Caiozzo}

\section{OpenEdition}

\section{Journals}

Édition électronique

URL : https://journals.openedition.org/medievales/6176

DOI : 10.4000/medievales.6176

ISSN : 1777-5892

Éditeur

Presses universitaires de Vincennes

\section{Édition imprimée}

Date de publication : 31 décembre 2010

Pagination : 195-197

ISBN : 978-2-84292-267-2

ISSN : 0751-2708

\section{Référence électronique}

Anna Caiozzo, «Abolala soudavar, The Aura of Kings, Legitimacy and Divine Sanction in Iranian Kingship, Mazda Publisher, Costa Mesa, 2003, 178 p. », Médiévales [En ligne], 59 | automne 2010, mis en ligne le 10 mars 2011, consulté le 22 avril 2022. URL : http://journals.openedition.org/medievales/6176 ; DOI : https://doi.org/10.4000/medievales.6176

Ce document a été généré automatiquement le 22 avril 2022.

Tous droits réservés 


\title{
Abolala SOUDAVAR, The Aura of Kings, Legitimacy and Divine Sanction in Iranian Kingship, Mazda Publisher, Costa Mesa, 2003, 178 p.
}

\author{
Anna Caiozzo
}

1 En 2003, Abolala Soudavar présentait un petit ouvrage sur La Gloire des rois, Légitimité et sanction divine dans la monarchie iranienne. Dès son introduction, l'auteur n'hésitait pas à prévenir le lecteur du caractère polémique du propos développé dans ce livre, qui fut à l'origine un article refusé par une revue iranisante bien connue, en raison d'une partie de l'argumentation reposant sur les thèses contestées de A. D. H. Bivar relatives au mithriacisme ésotérique ${ }^{1}$.

2 Pourtant, l'auteur ne se découragea pas et fit de l'article un volume qui mérite d'être remarqué par son propos novateur relatif à la Gloire ou Splendeur divine, pour reprendre le titre d'un ouvrage bien connu d'Helena Cassin et que de nombreux autres travaux sur le sujet émanant d'iranologues notoires évoquent sporadiquement depuis un demi-siècle : en effet, la Gloire, un concept évoquant l'essence même du pouvoir dans le monde iranien, non seulement survécut à l'époque islamique, mais s'exprima de façon très diversifiée dans la miniature orientale puis moghole.

3 Abolala Soudavar n'hésite pas en effet à montrer combien l'étude de l'image dans les manuscrits à peintures de l'Orient médiéval peut s'avérer fructueuse, et ce contrairement à bien des idées reçues sur la question. L'exploitation des miniatures, leur lecture polysémique, le rôle qu'elles jouent, tant dans la perception des idéologies politiques que des pratiques culturelles et anthropologiques, sont en effet sous-estimés par une grande partie des orientalistes contemporains. L'image en général, et la miniature en particulier, est appréhendée de façon timorée, remplie de préjugés sur le rôle qu'elle est supposée jouer, souvent limité à des fins décoratives; c'est cet a priori que l'historien de demain devra dépasser pour comprendre le rôle exact des images et 
leurs fonctions dans le monde islamique médiéval, en particulier dans l'aire turcomongole.

4 Les universitaires américains, P. Soucek, S. Blair, E. Sims ${ }^{2}$ ont ces vingt dernières années rédigé d'éloquents articles sur l'usage du portrait, du jardin, des fresques, etc. dans les miniatures commanditées par les princes timourides. Moins frileux que les Européens, ils osent enfin proposer un sens et une interprétation à la miniature orientale. C'est aussi l'objet de la démarche d'A. Soudavar dans cette étude sur le khvarna préislamique, encore appelé farr dans le monde musulman, qualité dont sont investis les souverains et qui fut longuement définie en 1943 par H. W. Bailey ${ }^{3}$, puis précisée par la plupart des spécialistes du monde iranien dont G. Gnoli ou J. DuchesneGuillemin ${ }^{4}$.

5 En sept chapitres, A. Soudavar expose tous les symboles constituant d'après lui les éléments visibles de la Gloire divine qui pare de son éclat, d'après les mythes fondateurs, les rois mythiques de l'Iran et ceux plus historiques - achéménides, parthes ou sassanides - qui firent sculpter pour la postérité des bas-reliefs représentant leur investiture par les dieux et leurs triomphes.

6 L'auteur montre comment un certain nombre de symboles de la Gloire, hérités en grande partie du monde égyptien puis transmis au monde mésopotamien, parvinrent dans le monde iranien zoroastrien, voire pré-zoroastrien, pour signifier que le souverain digne de régner sur les hommes et le monde était le dépositaire d'un élément d'essence divine le guidant dans la victoire. La Gloire royale, souvent matérialisée dans les bas-reliefs préislamiques par une aura encerclant la tête des rois, est cependant symbolisée par divers autres signes: shamsa (aura), dastâr (mouchoir), sarpech (aigrette), ailes de faucon, lotus ou des éléments animaliers variés (cornes de bélier, lion et taureau, ailes associés au scorpion, etc.).

7 Certains de ces symboles sont associés dans les croyances zoroastriennes à une divinité, voire en indiquent plusieurs, comme le lotus ou Apam-Napât, esprit des eaux associé à Anâhita ou à Mithra. La démonstration est souvent séduisante à l'image des variations du farr; quant au lotus, certes visible sur de nombreux supports, il ne doit pas faire oublier le roseau qui recueillit la Gloire enfuie de Jamshîd... Le cas du dastâr (mouchoir) est sans nul doute le plus intéressant car il associe la couronne enrubannée (à l'origine de lauriers ou d'olivier), portée par les victoires (un héritage iconographique grec et romain), au ciel tendu au-dessus des rois et à l'anneau d'investiture remis par les dieux. Cet anneau "magique» à lui seul est pourtant symbolique d'une puissance royale d'origine "divine", si l'on pense au roi de Perse Tahmûrath qui en était titulaire, ou plus tard à Salomon qui en fut doté. Quoi qu'il en soit, la métamorphose du cercle et des rubans flottants constituant le diadème des rois parthes et sassanides en mouchoir (dastâr), tenu par les rois dans les miniatures, devenu symbole du khvarna, est certes quelque peu prosaïque mais incontestable. Quant au turban, en dépit de sa forte charge symbolique, il est peu porté par les rois dans les miniatures. Concernant les symboles animaux, on peut avoir une opinion partagée car, autant le lion est associé au pouvoir, y compris par ses implications astrologiques, autant les réminiscences des cultes mithriaques semblent peu convaincantes. Le sarpech, enfin, est seulement évoqué : estil seulement associé au khvarnah?

Concernant la shamsa, l'aura de Gloire des élus, certains historiens de l'art, tels R. Milstein ou A. S. Mélikian Chirvani ${ }^{5}$, ont bien montré le rôle de la lumière dans l'art islamique, en particulier comment ce symbole pare désormais les prophètes et les 
saints comme on peut le voir dans le Mirâj Nâmeh parisien (Paris, BnF, ms. sup. turc 190, Herat, 1436). En effet, dans le monde musulman, les seuls dépositaires de la volonté divine sont les prophètes, augmentés pour les chiites, des imams et des saints, et non les rois, exception faite pour le registre astrologique. La lune par exemple, dans le frontispice du Kitâb al-diryâq (Paris, BnF, ms. arabe 2964, Irak, 1199), est représentée comme un dynaste en majesté porteur d'une couronne décorée d'un oiseau, réminiscence probable des couronnes ailées et des ailes de faucon, symboles de Verethragna... En effet, la sécularisation du pouvoir royal dans le monde musulman ne gomme pas la volonté d'identification du roi ou de l'émir au souverain cosmique qu'il fut dans les temps préislamiques, comme on peut le voir dans le réemploi de thèmes astrologiques par les sultans saljoukides ou les émirs de Haute Mésopotamie (E. Whelan) ou les souverains ayyoubides ou mongols (E. Baer $\left.{ }^{6}\right)$. D'ailleurs, plus que le khvarna, c'est la royauté cosmique qui survit dans les manuscrits à peintures orientaux, à l'époque saljouqide et artouqide, à travers les représentations astrologiques ou les portiques célébrant les trônes cosmiques (chez Jazarî) ${ }^{7}$, puis à l'époque mongole avec des « innovations visuelles", tel le trône cosmique à sept degrés, ou l'oiseau (que l'on peut imaginer porteur du farr) qui décore parfois ces mêmes trônes dans certaines copies du Shâh Nâmeh (Londres, British Library, ms. Or. 12688, Iran, 1446).

9 Les legs politico-religieux des époques antérieures survivent donc dans la miniature aux côtés d'éléments nouveaux venant d'autres aires culturelles (le monde turcomongol). On peut certes apporter des restrictions à certaines des propositions d'Abolala Soudavar, mais tout en gardant présent à l'esprit la chance dont dispose l'historien face aux nombreux corpus imagés d'une grande richesse, et dont l'essentiel reste à déchiffrer.

\section{NOTES}

1. A. D. H. BIVAR, «The Royal Hunter and the Hunter God. Esoteric Mithriaism under the Sasanians? ", Res Orientales, VII, 1995, p. 29-38.

2. P. P. SOUCEK, "The Theory and Practise of Portraiture in the Persian Tradition », Muqarnas, 17, 2000, p. 97-108 ; S. S. BLAIR, "Timurid Signs of Sovereignety ", Oriente moderno, XV, 76, 2, 1996, p. 551-576 et E. SIMS, "Sultan Husayn Bayqara's Zafarnamah and its Miniatures », dans The Memorial Volume of the VIth International Congress of Iranian Art and Archeology, Oxford, 1972, Téhéran 1996, p. 299-311 ; ID., «Ibrahim-Sultan's Illustrated Zafarnama of 839/1436 », Islamic Art, 4, 1990-91, p.175-218; ID., The Garett Manuscript of the Zafar-Nāme: A Study in Fifteenth - Century Patronage, PhD dissertation, New York, Institute of Fine Arts, 1973; ID. "The Illustrated Manuscripts of Firdausī's Shahnāma commissionned by Princes of the House of Tīmūr ", Ars Orientalis, XX, 1992, p. 44-68.

3. H. W. BAILEY, Zoroastrian Problems in the Ninth-Century Books, Oxford, 1943.

4. G. GNOLI, « Note sullo xvaranah », dans Acta Iranica vol IX, Hommages et Opera Minora, Orientalia J. Duchesne-Guillemin Emerito Oblata, Leyde, 1984, p. 204-218 ; J. DUCHESNE-GUILLEMIN, « Le X'ar'enah », Annali del Istituto universitario Orientale, Sezione linguistica, Naples, 1962, p. 19-31. 
5. R. MILSTEIN, «Light, Fire and the Sun in Islamic Painting", Studies in Islamic History and Civilization in Honour of Prof. David Ayalon, M. SHARON éd., Jérusalem, Leyde, 1986, p. 533-552 ; A. S. MÉLIKIAN-CHIRVANI, «The Iranian Sun Shield », Bulletin of the Asia Institute, 6, 1992, p. 1-42.

6. E. BAER, «The Ruler in Cosmic Setting: A Note on Medieval Islamic Iconography », dans Essays in Islamic Art and Architecture, In Honor of K. Otto-Dorn, ABBAS DANESHVARI éd., Malibu, 1981, p. 13-19.

7. Al-JAZARî, Kitâb fi ma'rifat al-Hilal al-Handasiyya, trad. D. R. HILL, The Book of Knowledge of Ingenious Mechanical Devices, Boston-Dordrecht, 1974. 